\title{
A Comparative Assessment of the Perturbative and Renormalized Coupled Cluster Theories with a Non-Iterative Treatment of Triple Excitations for Thermochemical Kinetics, Including a Study of Basis Set and Core Correlation Effects
}

Jingjing Zheng, ${ }^{1}$ Jeffrey R. Gour, ${ }^{2}$ Jesse J. Lutz, ${ }^{2}$ Marta Włoch, ${ }^{2, \# ~}{ }^{1}$ Piotr Piecuch, $, 2,3,{ }^{*}$ and Donald G. Truhlar ${ }^{1, *}$

${ }^{1}$ Department of Chemistry and Supercomputing Institute, University of Minnesota, 207 Pleasant Street S.E. Minneapolis, MN 55455 USA

${ }^{2}$ Department of Chemistry, Michigan State University, East Lansing, MI 48824 USA

${ }^{3}$ Department of Physics and Astronomy, Michigan State University, East Lansing, MI 48824 USA

Abstract. The CCSD, CCSD(T), and CR-CC $(2,3)$ coupled cluster methods, combined with five triple-zeta basis sets, namely MG3S, aug-cc-pVTZ, aug-cc-pV(T+d)Z, aug-cc-pCVTZ, and aug-cc-pCV(T+d)Z, are tested against the $\mathrm{DBH} 24$ database of diverse reaction barrier heights. The calculations confirm that the inclusion of connected triple excitations is essential to achieving high accuracy for thermochemical kinetics. They show that various non-iterative ways of incorporating connected triple excitations in coupled cluster theory, including the $\operatorname{CSSD}(\mathrm{T})$ approach, the full CR-CC $(2,3)$ method, and approximate variants of CR-CC $(2,3)$ similar to the triples corrections of the $\operatorname{CCSD}(2)$ approaches, are all about equally accurate for describing the effects of connected triply excited clusters in studies of activation barriers. The effect of freezing core electrons on the results of the CCSD, $\operatorname{CCSD}(\mathrm{T})$, and CR-CC $(2,3)$ calculations for barrier heights is also examined. It is demonstrated that to include core correlation most reliably, a basis set including functions that correlate the core and that can treat core-valence correlation is required. On the other hand, the frozen-core approximation using valence-optimized basis sets that lead to relatively small computational costs of $\mathrm{CCSD}(\mathrm{T})$ and $\mathrm{CR}-\mathrm{CC}(2,3)$ calculations can achieve almost as high accuracy as the analogous fully correlated calculations.

\footnotetext{
\# Present address: Department of Chemistry, Michigan Technological University, 1400 Townsend Dr., Houghton, MI 49931, USA

*Corresponding authors:P.P (piecuch@chemistry.msu.edu) and D. G. T. (truhlar@umn.edu)
} 


\section{Introduction}

Coupled cluster theory ${ }^{1}$ has become a standard method for highly accurate molecular electronic structure calculations. The popularity of coupled cluster theory for accurate calculations is primarily associated with the success of the $\operatorname{CCSD}(\mathrm{T})^{2}$ method, which includes singly and doubly excited clusters by solving the CCSD (coupled cluster singles and doubles) ${ }^{3}$ equations and connected triply excited clusters through a non-iterative quasiperturbative correction added to the CCSD energy (CCSD already includes the disconnected triply excited clusters). $\operatorname{CCSD}(\mathrm{T})$ provides a size-extensive, well-balanced, and highly accurate description of many-electron correlation effects for systems dominated by dynamical correlation, and computational costs are manageable for small and moderate-sized systems because of the noniterative nature of the triples treatment. The popularity of the $\operatorname{CCSD}(\mathrm{T})$ method is also related to the fact that it is an easy-to-use single-reference approach. However, $\operatorname{CCSD}(\mathrm{T})$ can fail dramatically for describing systems involving larger nondynamical correlation effects, which arise when electronic near degeneracy ${ }^{4}$ is present. $^{5-8}$ At least for the situations involving single bond stretching or breaking, and reaction pathways involving biradicals, this problem is remedied or ameliorated to a large extent by one of the renormalized $\operatorname{CCSD}(\mathrm{T})$ methods, ${ }^{5-8}$ including the recently developed rigorously size extensive variant of the completely renormalized $\operatorname{CCSD}(\mathrm{T})$ theory, termed $\operatorname{CR}-\mathrm{CC}(2,3){ }^{6}$

The CR-CC(2,3) method has so far been tested mainly for systems with even numbers of electrons, ${ }^{6,7}$ where near degeneracy occurs in biradicals and dissociating (or very stretched) bonds. The recent extension of the CR-CC(2,3) approach to systems with odd numbers of electrons ${ }^{8}$ has enabled treating near degeneracy cases more broadly, and the present communication provides the first systematic comparison of the performance of CR$\mathrm{CC}(2,3)$ and $\operatorname{CCSD}(\mathrm{T})$ for a diverse collection of reaction barrier heights. A few different variants of the $\mathrm{CR}-\mathrm{CC}(2,3)$ theory are examined, including the full $\mathrm{CR}-\mathrm{CC}(2,3)$ approach 
developed in Ref. 6 and three approximate variants of CR-CC $(2,3)$ which are obtained by dropping terms in the full CR-CC(2,3) energy formula. ${ }^{6,8}$ Two of these variants are practically identical to the triples corrections of the $\operatorname{CCSD}(2)$ theories developed in Refs. 9 and 10 , giving us an opportunity to systematically examine the consistency among a larger variety of the non-iterative triples coupled cluster methods in the context of the activation barrier calculations.

Testing coupled cluster methods for calculating barrier heights against large databases, such as Database $/ 3^{11}$ and NHTBH38/04, ${ }^{12}$ is very time-consuming. To more efficiently assess the performance of theoretical methods, we developed a smaller representative benchmark suite for thermochemical kinetics, called DBH24. ${ }^{13}$ This representative benchmark suite statistically represents the full data set of all the forward and reverse barrier heights of the hydrogen transfer reactions (22 reactions) in Database/3 and the diverse reactions in NHTBH38/04 (19 reactions). It consists of four types of reactions, namely hydrogen transfer (HT), heavy-atom transfer (HAT), nucleophilic substitution (NS), and unimolecular and association (UA) reactions. There are 3 reactions (6 barrier heights because forward and reverse reactions are considered) for each type of reaction in the DBH24 database. The six barrier heights of each reaction type are denoted as HATBH6, NSBH6, UABH6, and HTBH6, respectively. This representative benchmark suite has been used to test over 200 methods (combinations of theory levels and basis sets). ${ }^{13}$ The theory levels tested in the earlier work included single-level wave function methods, such as Hartree-Fock theory, Møller-Plesset perturbation theory, quadratic configuration interaction approach, and coupled cluster theory; they also included multicoefficient correlation methods, local and hybrid density functional theory, and semiempirical molecular orbital methods.

In this paper, we focus on testing the $\mathrm{CCSD}, \mathrm{CCSD}(\mathrm{T})$, and $\mathrm{CR}-\mathrm{CC}(2,3)$ approaches, including four different variants of the $\mathrm{CR}-\mathrm{CC}(2,3)$ theory, using all of the reactions included 
in the DBH24 database and five different basis sets of the triple-zeta quality with and without a frozen-core approximation. One of the main objectives of the present work is to determine if the recently developed $\mathrm{CR}-\mathrm{CC}(2,3)$ methodology, which eliminates failures of $\operatorname{CCSD}(\mathrm{T})$ in the biradical and bond breaking situations, is as effective as the $\operatorname{CCSD}(\mathrm{T})$ approach in studies of barrier heights, where $\operatorname{CCSD}(\mathrm{T})$ is usually successful. If this turns out to be true, the $\mathrm{CR}-\mathrm{CC}(2,3)$ approach can serve as a potentially significant improvement over $\operatorname{CCSD}(\mathrm{T})$, since it is very useful to have a theory that preserves the characteristics of $\operatorname{CCSD}(\mathrm{T})$ when $\operatorname{CCSD}(\mathrm{T})$ works and that can overcome the deficiencies of $\operatorname{CCSD}(\mathrm{T})$ when $\operatorname{CCSD}(\mathrm{T})$ breaks down. Moreover, by comparing the results of the frozen-core and allelectron calculations, we examine how effective the frozen-core approximation is in calculations of barrier heights. The effect of freezing the core on the results of coupled cluster calculations for a diverse set of barrier heights included in the DBH24 database has not been systematically studied before. In particular, we analyze the importance of additional basis set functions that correlate core electrons and that can treat core-valence correlation effects in coupled-cluster calculations.

We examine the performance of the variety of coupled cluster methods in which connected triple excitations are included non-iteratively to see if different ways of handling the corrections due to connected triply excited clusters lead to a consistent description of a diverse set of reaction barrier heights. We focus on coupled cluster methods of the CCSD(T) and CR-CC(2,3)/CCSD(2) type, since higher-level coupled cluster approaches, such as CCSDT, ${ }^{14}$ and methods including connected quadruply excited clusters have much larger computer costs that limit their applicability to small few-electron systems. Indeed, full CCSDT calculations involve expensive iterative steps that scale as $n_{\mathrm{o}}{ }^{3} n_{\mathrm{u}}{ }^{5}$, where $n_{\mathrm{o}}$ and $n_{\mathrm{u}}$ are the numbers of occupied and unoccupied orbitals, respectively, that are used in post-SCF calculations. The least expensive corrections due to connected quadruples have steps that 
scale as $n_{\mathrm{o}}{ }^{4} n_{\mathrm{u}}{ }^{5}, n_{\mathrm{o}}{ }^{2} n_{\mathrm{u}}{ }^{5}$, or $n_{\mathrm{u}}{ }^{6}$ (see, e.g., Refs. 5, 9, and 10, and references therein for more details), which are prohibitive in applications where larger numbers of electrons and larger basis sets are employed. For comparison, the $\operatorname{CCSD}(\mathrm{T})$ and $\mathrm{CR}-\mathrm{CC}(2,3)$ methods, and the related connected triples corrections of the $\operatorname{CCSD}(2)$ type have relatively inexpensive steps that scale as $n_{\mathrm{o}}{ }^{2} n_{\mathrm{u}}{ }^{4}$ in the iterative CCSD part and $n_{\mathrm{o}}{ }^{3} n_{\mathrm{u}}{ }^{4}$ in the non-iterative triples part. In consequence, the $\operatorname{CCSD}(\mathrm{T})$ and $\mathrm{CR}-\mathrm{CC}(2,3)$ approaches can be routinely applied to systems with up to about 80-100 correlated electrons and hundreds of basis functions. Because of these various practical considerations, it is important to examine the relative performance of the $\operatorname{CCSD}(\mathrm{T})$ and $\mathrm{CR}-\mathrm{CC}(2,3)$ methods and the effect of freezing core electrons on the quality of barrier heights predicted by the $\operatorname{CCSD}(\mathrm{T})$ and $\mathrm{CR}-\mathrm{CC}(2,3)$ calculations, as is done in the present study.

\section{Computational Details}

In the present work we systematically assessed the performance of the most practical coupled cluster methods, including the $\operatorname{CSSD}, \operatorname{CCSD}(\mathrm{T})$, and $\mathrm{CR}-\mathrm{CC}(2,3)$ approaches, with five triple-zeta basis sets by either correlating all electrons or using the frozen-core ${ }^{15}$ approximation. The CCSD and $\operatorname{CCSD}(\mathrm{T})$ methods have been in wide use for about two decades and are, therefore, well established. The details of the more recent CR-CC $(2,3)$ theory can be found elsewhere as well. ${ }^{6,8}$ Here, we only mention that in analogy to the conventional $\operatorname{CCSD}(\mathrm{T})$ approach, in the $\mathrm{CR}-\mathrm{CC}(2,3)$ calculations we add a correction due to triply excited clusters to the CCSD energy. The difference between CCSD(T) and CR$\mathrm{CC}(2,3)$ lies in the definition of the connected triples correction, which in the $\mathrm{CR}-\mathrm{CC}(2,3)$ case uses the complete form of the triply excited moments of the CCSD equations (projections of the CCSD equations on triply excited determinants) rather than the leading contributions to these moments used in $\operatorname{CCSD}(\mathrm{T})$. Moreover, in the $\mathrm{CR}-\mathrm{CC}(2,3)$ approach, 
one renormalizes the connected triples correction through the use of the left eigenstate of the similarity transformed Hamiltonian of coupled cluster theory, which adds the necessary flexibility in handling the biradical and bond breaking regions of molecular potential energy surfaces.

The CR-CC $(2,3)$ triples correction is defined, in particular, through the diagonal matrix elements $\left\langle\Phi_{i j k}^{a b c}\left|\bar{H}^{C C S D}\right| \Phi_{i j k}^{a b c}\right\rangle$, which enter the relevant perturbative energy denominator. Here, $\bar{H}^{C C S D}=e^{-T_{1}-T_{2}} H e^{T_{1}+T_{2}}$ is the similarity-transformed Hamiltonian of CCSD, with $T_{1}$ and $T_{2}$ representing the CCSD singly and doubly excited cluster operators, and $\left|\Phi_{i j k}^{a b c}\right\rangle$ are the triply excited determinants. ${ }^{6,8}$ The full CR-CC(2,3) method, in which no terms in $\left\langle\Phi_{i j k}^{a b c}\left|\bar{H}^{C C S D}\right| \Phi_{i j k}^{a b c}\right\rangle$ are neglected, is referred to as variant D of CR-CC(2,3), labeled as CR-CC(2,3),D. By dropping the three-body contributions in $\left\langle\Phi_{i j k}^{a b c}\left|\bar{H}^{C C S D}\right| \Phi_{i j k}^{a b c}\right\rangle$ (i.e., by retaining one- and two-body contributions only), we obtain variant $\mathrm{C}$ of $\mathrm{CR}-\mathrm{CC}(2,3)$, designated as $\mathrm{CR}-\mathrm{CC}(2,3), \mathrm{C}$. By retaining only the one-body contributions to $\left\langle\Phi_{i j k}^{a b c}\left|\bar{H}^{C C S D}\right| \Phi_{i j k}^{a b c}\right\rangle$, we obtain variant B, labeled as CR-CC(2,3),B. Finally, by replacing the one-body terms in $\left\langle\Phi_{i j k}^{a b c}\left|\bar{H}^{C C S D}\right| \Phi_{i j k}^{a b c}\right\rangle$ by the usual orbital energy differences $\left(\varepsilon_{a}+\varepsilon_{b}+\varepsilon_{c}-\varepsilon_{i}-\varepsilon_{j}-\varepsilon_{k}\right)$, while neglecting other many-body terms in $\left\langle\Phi_{i j k}^{a b c}\left|\bar{H}^{C C S D}\right| \Phi_{i j k}^{a b c}\right\rangle$, we obtain variant $\mathrm{A}$, designated as $\mathrm{CR}-\mathrm{CC}(2,3)$, A.

Variants A and B of the $\mathrm{CR}-\mathrm{CC}(2,3)$ approach are closely related to the triples parts of the $\operatorname{CSD}(2)$ corrections developed by Hirata et al. ${ }^{9}$ (variant A) and Head-Gordon et al. ${ }^{10}$ (variant B), as discussed in detail in Refs. 6 and 8. In particular, the CR-CC(2,3),A method is equivalent to the $\operatorname{CCSD}(2)_{\mathrm{T}}$ approach of Hirata et al., ${ }^{9}$ when canonical Hartree-Fock orbitals are used. The CR-CC(2,3),B approach is equivalent, up to small details, to the triples 
correction of the CCSD(2) method developed by Head-Gordon et al. ${ }^{10}$ As explained in Refs. 6 and 8 , the computer cost of each type of $\mathrm{CR}-\mathrm{CC}(2,3)$ calculations is approximately twice the cost of the conventional $\operatorname{CCSD}(\mathrm{T})$ calculations.

All calculations in this study are based on reactant, product, and transition structures optimized at the QCISD/MG3 level with the spin-restricted formalism for closed-shell systems and the fully spin-unrestricted formalism for open-shell systems. All of our experiences to date indicate that these geometries are well suited for the present study, although we plan to examine the effect of the geometries optimized at higher levels of theory on the quality of coupled cluster results reported in this work in the future study. The main purpose of the present study is to assess the reliability of various coupled cluster approaches, basis sets, and the frozen-core approximation when one uses the QCISD/MG3 geometries. The effect of spin-orbit coupling was added to the energies of the $\mathrm{Cl}$ and $\mathrm{OH}$ radicals, which lower their energies by 0.84 and $0.20 \mathrm{kcal} / \mathrm{mol}$, respectively.

The CCSD(T) calculations were performed using the MOLPRO 2002.6 package. $^{16}$ Spin-restricted Hartree-Fock calculations were used to obtain the reference orbitals with spin-unrestricted correlation calculations for open-shell systems and spin-restricted correlation calculations for closed-shell systems. The CR-CC(2,3),A-D calculations were carried out with the recently developed closed-shell ${ }^{6}$ and open-shell ${ }^{8} \mathrm{CR}-\mathrm{CC}(2,3)$ codes, which use the same choices as in MOLPRO for spin restriction and unrestriction and which have been incorporated in GAMESS. ${ }^{17}$ The underlying CCSD calculations were performed with both MOLPRO and GAMESS. In the case of GAMESS, the relevant CCSD routines were described in Ref. 18, for the closed-shell case, and Ref. 8, for the open-shell case.

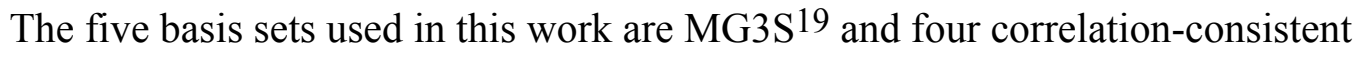
basis sets, namely, aug-cc-pVTZ, ${ }^{20,21}$ aug-cc-pV(T+d)Z, ${ }^{22}$ aug-cc-pCVTZ, ${ }^{20,23}$ and aug-cc$\mathrm{pCV}(\mathrm{T}+\mathrm{d}) \mathrm{Z}$. Note that MG3S is identical to $6-311+\mathrm{G}(3 \mathrm{~d} 2 \mathrm{f}, 2 \mathrm{df}, 2 \mathrm{p})$ for $\mathrm{H}-\mathrm{Si}$ and is similar to 
$6-311+(3 d 2 f)$, but improved ${ }^{24}$ for $P-A r$. The aug-cc-pV(T+d)Z basis set is the same as augcc-pVTZ except that it has a single extra $d$ function for the second row atoms from Al through Ar, and the other $d$ functions of aug-cc-pVTZ are also optimized for these atoms. In this work, we generate the aug-cc-pCV(T+d)Z basis set which is the same as aug-cc-pCVTZ basis set except that all valence $d$ functions are taken from aug-cc-pV(T+d)Z plus two $d$ functions describing inner shells are taken from aug-cc-pCVTZ. The sizes of the various basis sets are indicated in Table 1.

First we carried out calculations with all electrons correlated; these will be denoted as “full”. The MG3S, aug-cc-pVTZ, aug-cc-pCVTZ, and aug-cc-pCV(T+d)Z basis sets were used in the full calculations. The MG3S and aug-cc-pVTZ basis sets are valence-optimized, but we used them in full calculations to test the combination of full calculation and valenceoptimized basis set.

We also performed calculations that account only for valence correlation. These are sometimes called "frozen core", but here we denote them just by omitting "full". The MG3S, aug-cc-pVTZ, and aug-cc-pV(T+d)Z basis set were used in frozen-core calculations.

All of the above basis set choices are geared toward systematically assessing how significant the effect of freezing the core is on the activation barriers included in the DBH24 database. They also allow us to examine if using smaller triple zeta basis sets of the MG3S quality leads to acceptable accuracies and consistent results at various levels of coupled cluster theory.

\section{Results and Discussions}

The entire set of reaction barrier heights for the $\mathrm{DBH} 24$ database, as calculated with the $\mathrm{CCSD}, \mathrm{CCSD}(\mathrm{T})$, and $\mathrm{CR}-\mathrm{CC}(2,3), \mathrm{A}-\mathrm{D}$ approaches, combined with five triple-zeta basis sets, MG3S, aug-cc-pVTZ, aug-cc-pV(T+d)Z, aug-cc-pCVTZ, and aug-cc-pCV(T+d)Z, are 
given in the supplementary material. ${ }^{25}$ The calculated mean signed errors (MSEs) and mean unsigned errors (MUEs) of these methods are listed in Table 2-4.

With all five basis sets, the CCSD method overestimates all the reaction barrier heights except for the forward barrier height of reaction $\mathrm{HCN} \rightarrow \mathrm{HNC}$ with the aug-cc-pVTZ basis set. The mean unsigned errors of the CCSD method are around or above $2.0 \mathrm{kcal} / \mathrm{mol}$ for the DBH24 database. Especially for heavy-atom transfer reactions, the CCSD method gives quite large errors (above $2.9 \mathrm{kcal} / \mathrm{mol}$ ), which is much worse than many density functionals with either polarized double-zeta or polarized triple-zeta basis sets. ${ }^{12}$

The $\operatorname{CCSD}(\mathrm{T})$ and CR-CC(2,3),A-D methods improve the results dramatically by including connected triple excitations, especially in conjunction with augmented correlationconsistent basis sets. In general, there is a great degree of consistency among the $\operatorname{CCSD}(\mathrm{T})$ and $\mathrm{CR}-\mathrm{CC}(2,3), \mathrm{A}-\mathrm{D}$ data which implies that the remaining relatively small errors must be due to higher-than-triply excited clusters and higher angular momentum functions that are not included in the basis sets of the triple zeta quality. In particular, the mean unsigned errors of $\operatorname{CCSD}(\mathrm{T})$ with the MG3S basis set are about $0.9-1.0 \mathrm{kcal} / \mathrm{mol}$ both when correlating all electrons and when correlating only valence electrons, whereas the mean unsigned errors characterizing the $\operatorname{CCSD}(\mathrm{T})$ results for the augmented correlation-consistent basis sets vary between 0.4 and $0.6 \mathrm{kcal} / \mathrm{mol}$. Comparison of Table 2 and Table 3 shows that fully correlated $\operatorname{CCSD}(\mathrm{T})$ and $\mathrm{CR}-\mathrm{CC}(2,3)$ calculations generally give slightly better results than frozen-core calculations. The $\operatorname{CCSD}(\mathrm{T})($ full)/aug-cc-pCV(T+d)Z method gives the best results among all the tested methods with a mean unsigned error of only $0.44 \mathrm{kcal} / \mathrm{mol}$, although the overall accuracy of the CR-CC(2,3),A-D approaches is practically the same as that of $\operatorname{CCSD}(\mathrm{T})$, with only minimally higher mean unsigned errors by $0.02-0.14 \mathrm{kcal} / \mathrm{mol}$ compared to $\operatorname{CCSD}(\mathrm{T})$ when the most complete variant of $\mathrm{CR}-\mathrm{CC}(2,3)$, i.e., variant $\mathrm{D}$, is examined. On the other hand, for the nucleophilic substitution reactions, $\mathrm{CR}-\mathrm{CC}(2,3)$ performs better than 
$\operatorname{CCSD}(\mathrm{T})$, improving the mean unsigned errors by about $0.1-0.3 \mathrm{kcal} / \mathrm{mol}$ with all the tested basis sets.

For the reactions containing $\mathrm{S}$ and $\mathrm{Cl}$ atoms, the additional $d$ functions in the aug-cc$\mathrm{pV}(\mathrm{T}+\mathrm{d}) \mathrm{Z}$ basis sets improve the frozen-core $\operatorname{CCSD}(\mathrm{T})$ and $\mathrm{CR}-\mathrm{CC}(2,3)$ results by about $0.2-0.3 \mathrm{kcal} / \mathrm{mol}$ compared with the aug-cc-pVTZ basis sets (see Table 4). ${ }^{25}$ On the other hand, the aug-cc-pCV(T+d)Z basis sets give almost the same results as the corresponding aug-cc-pCVTZ basis sets at the all-electron $\operatorname{CCSD}(\mathrm{T})$ level.

Unlike CCSD, CCSD(T) sometimes underestimates barrier heights; in particular, of the 24 barrier heights in the $\mathrm{DBH} 24$ database, it underestimates seven (eight) barrier heights in frozen-core (full) calculations with MG3S basis set, 17 barrier heights in fully correlated calculations with the aug-cc-pVTZ basis sets, and 14 barrier heights with each of the other three correlation-consistent basis sets. The $\mathrm{CR}-\mathrm{CC}(2,3)$ approach may also underestimate barrier heights in some cases, although not as often as CCSD(T). In particular, of the 24 barrier heights in the DBH24 database, 8 barriers obtained with the aug-cc-pCVTZ basis sets and 11 barriers obtained with each of the aug-cc-pV(T+d)Z and aug-cc-pVTZ basis sets are underestimated by the CR-CC(2,3),D calculations.

Although CCSD(T)(full)/MG3S and CR-CC(2,3)(full)/MG3S calculations occasionally improve the accuracy by about $0.05 \mathrm{kcal} / \mathrm{mol}$ as compared to frozen core approximation, it is not recommended to use valence-optimized basis sets when including core and core-valence correlation since this is not only more expensive, but a potential source of problems. ${ }^{26}$ Note that the MG3S basis set is much smaller than the aug-cc-pVTZ basis set, as shown in Table 1, while providing high quality results. The combination of the MG3S basis set with the $\operatorname{CCSD}(\mathrm{T})$ or $\mathrm{CR}-\mathrm{CC}(2,3)$ methods is a good choice to balance the accuracy and computational costs in calculations for larger systems. 
The mean unsigned errors characterizing the $\mathrm{CR}-\mathrm{CC}(2,3)$ results listed in Tables 2 and 3 show that variants $\mathrm{C}$ and $\mathrm{D}$ of $\mathrm{CR}-\mathrm{CC}(2,3)$ give almost the same results, which indicates the negligible role of the three-body components in the $\left\langle\Phi_{i j k}^{a b c}\left|\bar{H}^{C C S D}\right| \Phi_{i j k}^{a b c}\right\rangle$ terms defining the $\mathrm{CR}-\mathrm{CC}(2,3)$ triples correction. They are also on average somewhat more accurate than the formally less complete A and B variants when the MG3S basis set is employed. Variants A and B of CR-CC $(2,3)$ seem to be slightly more accurate than variants $\mathrm{C}$ and D when the correlation-consistent basis sets are employed. The CR-CC(2,3) method, particularly its most complete variant $\mathrm{D}$, represents a useful alternative to $\operatorname{CCSD}(\mathrm{T})$, preserving, as shown here, the high accuracy of $\operatorname{CCSD}(\mathrm{T})$ in barrier height calculations, while offering significant advantages over $\operatorname{CCSD}(\mathrm{T})$ in the more multi-reference bond breaking and biradical regions examined in the earlier work. ${ }^{6-8}$

\section{Summary and Concluding Remarks}

We have tested six practical variants of coupled cluster theory with five types of basis sets against a diverse barrier height database. Our results are consistent with the earlier studies in the literature that indicate the inclusion of connected triple excitations is essential to achieving high accuracy for thermochemical kinetics with coupled cluster theory. We have provided additional extensive evidence in this regard by showing that the CCSD(T) method and four variants of the recently developed $\mathrm{CR}-\mathrm{CC}(2,3)$ theory, including two variants that are practically equivalent to the triples corrections of the $\operatorname{CCSD}(2)$ approaches, give very similar results for a wide variety of barrier heights in the DBH24 database. The observed consistency among various non-iterative triples coupled cluster levels indicates that the elimination of the remaining small errors in the $\operatorname{CCSD}(\mathrm{T})$ and $\mathrm{CR}-\mathrm{CC}(2,3)$ results would require using higher and much less practical levels of coupled cluster theory and basis sets larger than the triple zeta basis sets tested in the present work. We have systematically 
examined the effect of freezing core electrons and additional functions in a basis set that describe core correlations on the quality of coupled cluster results for a diverse set of activation barriers included in the DBH24 database. We have demonstrated that to include core correlation energy reliably in coupled cluster calculations, basis sets including functions that correlate the core and that can treat core-valence correlation, such as aug-cc-pCV $(\mathrm{T}+\mathrm{d}) \mathrm{Z}$, are required. On the other hand, the frozen-core approximation can achieve high accuracy using considerably smaller valence-optimized basis sets, such as MG3S, with relatively inexpensive computational costs. Our results indicate that on average the $\mathrm{CR}-\mathrm{CC}(2,3)$ theory provides activation barriers that are very similar to those obtained with the conventional $\mathrm{CCSD}(\mathrm{T})$ method, when the $\mathrm{DBH} 24$ database is examined. This is an encouraging finding, particularly considering the fact that the $\mathrm{CR}-\mathrm{CC}(2,3)$ approach is also capable of eliminating failures of $\operatorname{CCSD}(\mathrm{T})$ in the bond breaking and biradical regions of molecular potential energy surfaces, as shown, for example, in Refs. 6-8, without making the calculations more complicated or considerably more expensive. Based on the results provided in this paper and the earlier work reported in Refs. 6-8, we can conclude that the $\mathrm{CR}-\mathrm{CC}(2,3)$ approach can be regarded as an improvement over $\operatorname{CCSD}(\mathrm{T})$, since it is as accurate, on average, as $\mathrm{CCSD}(\mathrm{T})$ in calculations involving a diverse set of activation barriers constituting the DBH24 database, while overcoming the deficiencies of $\operatorname{CCSD}(\mathrm{T})$ in applications involving single bond breaking and biradicals.

\section{Acknowledgment}

The authors are grateful to Steven Mielke for helpful discussion. The work at the University of Minnesota was supported in part by the U. S. Department of Energy, Office of Basic Energy Sciences, under grant No. DE-FG02-86ER13579 (D.G.T.). The work at Michigan State University was supported by the U. S. Department of Energy, Office of Basic 
Energy Sciences, under grant No. DE-FG02-01ER15228 (PP), and by the National

Science Foundation's Graduate Research Fellowship (J.R.G.). Some of the calculations were performed on the computer systems provided by the High Performance Computing Center at Michigan State University. 


\section{References}

$1 \quad$ F. Coester, Nucl. Phys. 7, 421 (1958); F. Coester and H. Kümmel, Nucl. Phys. 17, 477 (1960); J. Čížek, J. Chem. Phys. 45, 4256 (1966); J. Č́ížek, Adv. Chem. Phys. 14, 35 (1969); J. Čížek and J. Paldus, Int. J. Quantum Chem. 5, 359 (1971); J. Paldus, J. Čížek, and I. Shavitt, Phys. Rev. A 5, 50 (1972); J. A. Pople, R. Krishnan, H. B. Schlegel, and J. S. Binkley, Int. J. Quantum Chem. 14, 545 (1978); R. J. Bartlett and G. D. Purvis, Int. J. Quantum Chem. 14, 561 (1978); R. J. Bartlett, J. Phys. Chem. 93, 1697 (1989); K. Raghavachari, Annu. Rev. Phys. Chem. 42, 615 (1991); R.J. Bartlett, in Modern Electronic Structure Theory, Part I, edited by D.R. Yarkony (World Scientific, Singapore, 1995), p. 1047; K. Raghavachari and J. B. Anderson, J. Phys. Chem. 100, 12960 (1996); J. Gauss, in Encyclopedia of Computational Chemistry, Vol. 1, edited by P. v. R. Schleyer, N. L. Allinger, T. Clark, J. Gasteiger, P. A. Kollman, H. F. Schaefer III, and P. R. Schreiner (Wiley, Chichester, 1998), p. 615; J. Paldus and X. Li, Adv. Chem. Phys. 110, 1 (1999); T. D. Crawford and H. F. Schaefer III, Rev. Comput. Chem. 14, 33 (2000); J. Paldus, in Handbook of Molecular Physics and Quantum Chemistry, Vol 2, edited by S. Wilson (Wiley, Chichester, 2003), p. 272.

2 K. Raghavachari, G. W. Trucks, J. A. Pople, and M. Head-Gordon, Chem. Phys. Lett. 157, 479 (1989).

3 G. D. Purvis, III and R. J. Bartlett, J. Chem. Phys. 76, 1910 (1982).

4 D. R. Hartree, W. Hartree, and B. Swirles, Philos. Trans. Roy. Soc. A 238, 229 (1939); V. McKoy and O. Sinanoglu, J. Chem. Phys. 41, 2689 (1964); G. Das and A. C. Wahl, J. Chem. Phys. 44, 87 (1966); J. H. Davis, W. A. Goddard, and L. B. Harding, J. Am. Chem. Soc. 99, 2919 (1977); P. Baybutt, F. W. Bobrowicz, L. R. Kahn, and D. G. Truhlar, J. Chem. Phys. 68, 4809 (1978); M. W. Schmidt and M. S. Gordon, Annu. Rev. Phys. Chem. 49, 233 (1998); D. G. Truhlar, J. Comput. Chem. 28, 73 (2007).

5 P. Piecuch and K. Kowalski, in Computational Chemistry: Reviews of Current Trends, edited by J. Leszczyński (World Scientific, Singapore, 2000), Vol. 5, p. 1; K. Kowalski and P. Piecuch, J. Chem. Phys. 113, 18 (2000); P. Piecuch, K. Kowalski, I. S. O. Pimienta and M. J. McGuire, Int. Rev. Phys. Chem. 21, 527 (2002); P. Piecuch, K. Kowalski, I. S. O. Pimienta, P.-D. Fan, M. Lodriguito, M. J. McGuire, S. A. Kucharski, T. Kuś, and M. Musiał, Theor. Chem. Acc. 112, 349 (2004). 
6 P. Piecuch and M. Włoch, J. Chem. Phys. 123, 224105 (2005); P. Piecuch, M. Włoch, J. R. Gour, and A. Kinal, Chem. Phys. Lett. 418, 467 (2006); M. Włoch, M. D.

Lodriguito, P. Piecuch, and J. R. Gour, Mol. Phys. 104, 2149 (2006).

7 C. J. Cramer, M. Włoch, P. Piecuch, C. Puzzarini, and L. Gagliardi, J. Phys. Chem. A 110, 1991 (2006); C. J. Cramer, A. Kinal, M. Włoch, P. Piecuch, and L. Gagliardi, J. Phys. Chem. A 110, 11557 (2006); A. Kinal and P. Piecuch, J. Phys. Chem. A 111, 734 (2007); P. Piecuch, M. Włoch, and A.J.C. Varandas, Theor. Chem. Acc., in press (2007), DOI: 10.1007/s00214-007-0297-3; Y. Ge, M.S. Gordon, and P. Piecuch, J. Chem. Phys. 127, 174106 (2007).

$8 \quad$ M. Włoch, J. R. Gour, and P. Piecuch, J. Phys. Chem. A 111, 11359 (2007).

9 S. Hirata, M. Nooijen, I. Grabowski, and R. J. Bartlett, J. Chem. Phys. 114, 3919 (2001); 115, 3967 (2001) (Erratum); S. Hirata, P.-D. Fan, A. A. Auer, M. Nooijen, and P. Piecuch, J. Chem. Phys. 121, 12197 (2004).

10 S. R. Gwaltney and M. Head-Gordon, Chem. Phys. Lett. 323, 21 (2000); S.R. Gwaltney, C.D. Sherrill, M. Head-Gordon, and A.I. Krylov, J. Chem. Phys. 113, 3548 (2000); S. R. Gwaltney and M. Head-Gordon, J. Chem. Phys. 115, 2014 (2001); S. R. Gwaltney, E. F. C. Byrd, T. Van Voorhis, and M. Head-Gordon, Chem. Phys. Lett. 353, 359 (2002).

11 B. J. Lynch and D. G. Truhlar, J. Phys. Chem. A 107, 3898 (2003).

12 Y. Zhao, N. Gonzalez-Garcia, and D. G. Truhlar, J. Phys. Chem. A 109, 2012 (2005).

13 J. Zheng, Y. Zhao, and D. G. Truhlar, J. Chem. Theory Comput. 3, 569 (2007).

14 J. Noga and R.J. Bartlett, J. Chem. Phys. 86, 7041 (1987); 89, 3401 (1988) (Erratum); G.E. Scuseria and H.F. Schaefer III, Chem. Phys. Lett. 152, 382 (1988).

E. S. Sachs, J. Hinze, and N. H. Sabelli, J. Chem. Phys. 62, 3393 (1975).

16 H.-J. Werner, P. J. Knowles, R. D. Amos, A. Bernhardsson, A. Berning, P. Celani, D. L. Cooper, M. J. O. Deegan, A. J. Dobbyn, F. Eckert, C. Hampel, G. Hetzer, T. Korona, R. Lindh, A. W. Lloyd, S. J. McNicholas, F. R. Manby, W. Meyer, M. E. Mura, A. Nicklass, P. Palmieri, R. Pitzer, G. Rauhut, M. Schtüz, U. Schumann, H. Stoll, A. J. Stone, R. Tarroni, and T. Thorsteinsson, MOLPRO (University of Birmingham, Birmingham, 2002).

17 M. W. Schmidt, K. K. Baldridge, J. A. Boatz, S. T. Elbert, M. S. Gordon, J. H. Jensen, S. Koseki, N. Matsunaga, K. A. Nguyen, S. Su, T. L. Windus, M. Dupuis, and J. A. Montgomery, J. Comput. Chem 14, 1347 (1993); M. S. Gordon and M. W. Schmidt, in Theory and Applications of Computational Chemistry: the first forty years, edited by C. 
E. Dykstra, G. Frenking, K. S. Kim, and G. E. Scuseria (Elsevier, Amsterdam, 2005), pp. 1167. These methods are available as of the June 2007 version. (2002).

19 B. J. Lynch, Y. Zhao, and D. G. Truhlar, J. Phys. Chem. A 107, 1384 (2003).

20 T. H. Dunning, J. Chem. Phys. 90, 1007 (1989); D. E. Woon and T. H. Dunning, J. Chem. Phys. 98, 1358 (1993).

21 R. A. Kendall, T. H. Dunning, and R. J. Harrison, J. Chem. Phys. 96, 6796 (1992).

22 T. H. Dunning, K. A. Peterson, and A. K. Wilson, J. Chem. Phys. 114, 9244 (2001).

23 D. E. Woon and T. H. Dunning, J. Chem. Phys. 103, 4572 (1995); K. A. Peterson and T. H. Dunning, J. Chem. Phys. 117, 10548 (2002).

24 L. A. Curtiss, K. Raghavachari, C. Redfern, V. Rassolov, and J. A. Pople, J. Chem. Phys. 109, 7764 (1998).

25 See EPAPS Document No. 000 for electronic files, in pdf format, containing tabulations of full set of calculated reaction barrier heights for the DBH24 database with CCSD, CCSD(T), and CR-CC(2,3) combined with aug-cc-pCVTZ, aug-cc-pVTZ, and MG3S basis sets. This document can be reached through a direct link in the online articles's HTML reference section or via the EPAPS homepage (http:// Www.aip.org/pubservs/epaps.html).

26 P. R. Taylor, in Lecture Notes in Quantum Chemistry, edited by B. O. Roos (SpringerVerlag, Berlin, 1992), pp. 406. 
Table 1. The numbers of primitive and contracted basis functions for the 12 transition states studied in this work. ${ }^{\mathrm{a}}$

\begin{tabular}{|c|c|c|c|c|c|}
\hline \multirow[b]{2}{*}{ Transition state } & \multicolumn{5}{|c|}{ Basis set } \\
\hline & MG3S & AVTZ & $\mathrm{AV}(\mathrm{T}+\mathrm{d}) \mathrm{Z}$ & ACVTZ & $\mathrm{ACV}(\mathrm{T}+\mathrm{d}) \mathrm{Z}$ \\
\hline $\mathrm{Cl}^{-} \ldots \mathrm{CH}_{3} \mathrm{Cl} \rightarrow \mathrm{ClCH}_{3} \ldots \mathrm{Cl}^{-}$ & $(249)[169]$ & $(316)[215]$ & $(328)[225]$ & $(390)[278]$ & $(402)[288]$ \\
\hline $\mathrm{CH}_{3}+\mathrm{FCl} \rightarrow \mathrm{CH}_{3} \mathrm{~F}+\mathrm{Cl}$ & $(219)[149]$ & $(299)[211]$ & $(305)[216]$ & $(357)[262]$ & $(363)[267]$ \\
\hline $\mathrm{F}^{-} \ldots \mathrm{CH}_{3} \mathrm{Cl} \rightarrow \mathrm{FCH}_{3} \ldots \mathrm{Cl}^{-}$ & $(219)[149]$ & $(299)[211]$ & $(305)[216]$ & $(357)[262]$ & $(363)[267]$ \\
\hline $\mathrm{OH}^{-}+\mathrm{CH}_{3} \mathrm{~F} \rightarrow \mathrm{HOCH}_{3}+\mathrm{F}^{-}$ & $(200)[138]$ & $(309)[230]$ & $(309)[230]$ & $(351)[269]$ & $(351)[269]$ \\
\hline $\mathrm{H}+\mathrm{C}_{2} \mathrm{H}_{4} \rightarrow \mathrm{CH}_{3} \mathrm{CH}_{2}$ & $(159)[113]$ & $(269)[207]$ & $(269)[207]$ & $(297)[233]$ & $(297)[233]$ \\
\hline $\mathrm{OH}+\mathrm{CH}_{4} \rightarrow \mathrm{CH}_{3}+\mathrm{H}_{2} \mathrm{O}$ & $(159)[113]$ & $(269)[207]$ & $(269)[207]$ & $(297)[233]$ & $(297)[233]$ \\
\hline $\mathrm{H}+\mathrm{N}_{2} \mathrm{O} \rightarrow \mathrm{OH}+\mathrm{N}_{2}$ & $(167)[111]$ & $(228)[161]$ & $(228)[161]$ & $(270)[200]$ & $(270)[200]$ \\
\hline $\mathrm{H}+\mathrm{H}_{2} \mathrm{~S} \rightarrow \mathrm{H}_{2}+\mathrm{HS}$ & $(115)[81]$ & $(165)[119]$ & $(171)[124]$ & $(195)[144]$ & $(201)[149]$ \\
\hline $\mathrm{H}+\mathrm{N}_{2} \rightarrow \mathrm{HN}_{2}$ & $(115)[77]$ & $(161)[115]$ & $(161)[115]$ & $(189)[141]$ & $(189)[141]$ \\
\hline $\mathrm{HCN} \rightarrow \mathrm{HNC}$ & $(115)[77]$ & $(161)[115]$ & $(161)[115]$ & $(189)[141]$ & $(189)[141]$ \\
\hline $\mathrm{H}+\mathrm{ClH} \rightarrow \mathrm{HCl}+\mathrm{H}$ & $(104)[72]$ & $(138)[96]$ & $(144)[101]$ & $(168)[121]$ & $(174)[126]$ \\
\hline $\mathrm{H}+\mathrm{OH} \rightarrow \mathrm{O}+\mathrm{H}_{2}$ & $(74)[52]$ & $(121)[92]$ & $(121)[92]$ & $(135)[105]$ & $(135)[105]$ \\
\hline Average & $(158)[108]$ & $(228)[165]$ & $(231)[167]$ & (266)[199] & $(269)[202]$ \\
\hline
\end{tabular}

a $(M)[N]$ denotes $M$ primitive functions and $N$ contracted functions. Furthermore, A is shorthand for aug-cc-p in the names of the augmented correlation consistent basis sets. 
Table 2. Mean signed error (MSE) and mean unsigned error (MUE) of coupled cluster methods calculated with all electrons correlated against DBH24 database (in $\mathrm{kcal} / \mathrm{mol})$.

\begin{tabular}{|c|c|c|c|c|c|c|c|c|c|}
\hline \multirow[t]{2}{*}{ Method } & \multicolumn{2}{|c|}{ HATBH6 } & \multicolumn{2}{|c|}{ NSBH6 } & \multicolumn{2}{|c|}{ UABH6 } & \multicolumn{2}{|c|}{ HTBH6 } & \multirow{2}{*}{$\begin{array}{r}\text { DBH24 } \\
\text { MUE }\end{array}$} \\
\hline & MSE & MUE & MSE & MUE & MSE & MUE & MSE & MUE & \\
\hline \multicolumn{10}{|c|}{ MG3S } \\
\hline CCSD(full) & 4.36 & 4.36 & 2.29 & 2.29 & 1.76 & 1.76 & 2.55 & 2.55 & 2.74 \\
\hline $\operatorname{CCSD}(\mathrm{T})($ full) & 0.92 & 1.24 & -0.01 & 0.74 & 0.70 & 0.70 & 0.93 & 1.04 & 0.93 \\
\hline CR-CC(2,3), A(full) & 1.50 & 1.61 & 0.27 & 0.54 & 0.93 & 0.93 & 1.13 & 1.14 & 1.06 \\
\hline CR-CC(2,3), B(full) & 1.72 & 1.77 & 0.47 & 0.57 & 0.96 & 0.96 & 1.19 & 1.19 & 1.12 \\
\hline CR-CC(2,3), C(full) & 1.16 & 1.35 & 0.10 & 0.59 & 0.82 & 0.82 & 0.98 & 1.04 & 0.95 \\
\hline CR-CC(2,3), D(full) & 1.17 & 1.35 & 0.10 & 0.60 & 0.82 & 0.82 & 0.98 & 1.04 & 0.95 \\
\hline \multicolumn{10}{|c|}{ aug-cc-pVTZ } \\
\hline CCSD(full) & 2.85 & 2.85 & 1.83 & 1.83 & 1.28 & 1.28 & 1.06 & 1.06 & 1.76 \\
\hline $\operatorname{CCSD}(\mathrm{T})($ full) & -0.72 & 0.84 & -0.52 & 0.64 & 0.17 & 0.34 & -0.72 & 0.72 & 0.64 \\
\hline \multicolumn{10}{|c|}{ aug-cc-pCVTZ } \\
\hline CCSD(full) & 3.61 & 3.61 & 2.12 & 2.12 & 1.13 & 1.13 & 1.75 & 1.75 & 2.15 \\
\hline $\operatorname{CCSD}(\mathrm{T})($ full) & -0.03 & 0.61 & -0.26 & 0.46 & 0.01 & 0.28 & -0.05 & 0.45 & 0.45 \\
\hline CR-CC(2,3), A(full) & 0.55 & 0.84 & 0.04 & 0.34 & 0.23 & 0.38 & 0.14 & 0.54 & 0.52 \\
\hline CR-CC(2,3), B(full) & 0.77 & 0.97 & 0.23 & 0.34 & 0.26 & 0.41 & 0.22 & 0.56 & 0.57 \\
\hline CR-CC(2,3), C(full) & 0.42 & 0.76 & -0.22 & 0.63 & 0.22 & 0.43 & 0.11 & 0.55 & 0.59 \\
\hline CR-CC(2,3), D(full) & 0.42 & 0.76 & -0.22 & 0.62 & 0.22 & 0.43 & 0.11 & 0.55 & 0.59 \\
\hline \multicolumn{10}{|c|}{ aug-cc-pCV(T+d)Z } \\
\hline CCSD(full) & 3.60 & 3.60 & 2.15 & 2.15 & 1.13 & 1.13 & 1.76 & 1.76 & 2.16 \\
\hline $\operatorname{CCSD}(\mathrm{T})($ full) & -0.05 & 0.58 & -0.24 & 0.44 & 0.01 & 0.28 & -0.05 & 0.45 & 0.44 \\
\hline
\end{tabular}


Table 3. Mean signed error (MSE) and mean unsigned error (MUE) of coupled cluster methods calculated with frozen core approximation against DBH24 database (in $\mathrm{kcal} / \mathrm{mol}$ ).

\begin{tabular}{|c|c|c|c|c|c|c|c|c|c|}
\hline \multirow[t]{2}{*}{ Method } & \multicolumn{2}{|c|}{ HATBH6 } & \multicolumn{2}{|c|}{ NSBH6 } & \multicolumn{2}{|c|}{ UABH6 } & \multicolumn{2}{|c|}{ HTBH6 } & \multirow{2}{*}{$\begin{array}{r}\text { DBH24 } \\
\text { MUE }\end{array}$} \\
\hline & MSE & MUE & MSE & MUE & MSE & MUE & MSE & MUE & \\
\hline \multicolumn{10}{|c|}{ MG3S } \\
\hline CCSD & 4.43 & 4.43 & 2.03 & 2.03 & 1.58 & 1.58 & 2.62 & 2.62 & 2.67 \\
\hline $\operatorname{CCSD}(\mathrm{T})$ & 1.06 & 1.37 & -0.25 & 0.94 & 0.53 & 0.53 & 1.04 & 1.10 & 0.98 \\
\hline CR-CC(2,3), A & 1.63 & 1.76 & 0.03 & 0.75 & 0.76 & 0.76 & 1.23 & 1.23 & 1.12 \\
\hline CR-CC(2,3), B & 1.85 & 1.91 & 0.22 & 0.63 & 0.80 & 0.80 & 1.29 & 1.29 & 1.16 \\
\hline CR-CC(2,3), C & 1.28 & 1.49 & -0.17 & 0.83 & 0.66 & 0.66 & 1.08 & 1.10 & 1.02 \\
\hline CR-CC(2,3), D & 1.29 & 1.49 & -0.18 & 0.83 & 0.65 & 0.65 & 1.08 & 1.10 & 1.02 \\
\hline \multicolumn{10}{|c|}{ aug-cc-pVTZ } \\
\hline CCSD & 3.54 & 3.54 & 1.66 & 1.66 & 1.03 & 1.11 & 1.72 & 1.72 & 2.01 \\
\hline $\operatorname{CCSD}(\mathrm{T})$ & 0.01 & 0.91 & -0.67 & 0.68 & -0.06 & 0.40 & -0.04 & 0.57 & 0.64 \\
\hline CR-CC(2,3), A & 0.58 & 1.20 & -0.39 & 0.44 & 0.17 & 0.47 & 0.15 & 0.62 & 0.68 \\
\hline CR-CC(2,3), B & 0.80 & 1.32 & -0.20 & 0.35 & 0.19 & 0.49 & 0.23 & 0.65 & 0.70 \\
\hline CR-CC(2,3), C & 0.46 & 1.13 & -0.70 & 0.77 & 0.16 & 0.48 & 0.11 & 0.64 & 0.75 \\
\hline CR-CC(2,3), D & 0.46 & 1.13 & -0.71 & 0.77 & 0.15 & 0.48 & 0.11 & 0.64 & 0.75 \\
\hline \multicolumn{10}{|c|}{ aug-cc-pV(T+d)Z } \\
\hline CCSD & 3.41 & 3.41 & 1.82 & 1.82 & 1.03 & 1.11 & 1.69 & 1.69 & 2.01 \\
\hline $\operatorname{CCSD}(\mathrm{T})$ & -0.13 & 0.67 & -0.53 & 0.62 & -0.06 & 0.40 & -0.06 & 0.54 & 0.56 \\
\hline CR-CC(2,3), A & 0.45 & 0.88 & -0.24 & 0.39 & 0.17 & 0.47 & 0.13 & 0.60 & 0.58 \\
\hline CR-CC $(2,3), \mathrm{B}$ & 0.67 & 1.00 & -0.05 & 0.30 & 0.19 & 0.49 & 0.20 & 0.63 & 0.61 \\
\hline CR-CC(2,3), C & 0.30 & 0.80 & -0.53 & 0.60 & 0.16 & 0.48 & 0.09 & 0.62 & 0.62 \\
\hline CR-CC(2,3), D & 0.31 & 0.80 & -0.54 & 0.60 & 0.15 & 0.48 & 0.09 & 0.62 & 0.62 \\
\hline
\end{tabular}


Table 4. Mean signed error (MSE) and mean unsigned error (MUE) for 5 reactions containing $\mathrm{S}$ and $\mathrm{Cl}$ (in $\mathrm{kcal} / \mathrm{mol}$ ).

\begin{tabular}{|c|c|c|c|c|c|c|c|}
\hline \multirow[t]{2}{*}{ Method } & \multicolumn{2}{|c|}{ SClHATBH4 } & \multicolumn{2}{|c|}{ SCINSBH4 } & \multicolumn{2}{|c|}{ SClHTBH2 } & \multirow{2}{*}{$\begin{array}{c}\text { SClDBH10 } \\
\text { MUE }\end{array}$} \\
\hline & MSE & MUE & MSE & MUE & MSE & MUE & \\
\hline \multicolumn{8}{|c|}{ aug-cc-pVTZ } \\
\hline CCSD & 2.77 & 2.77 & 1.28 & 1.28 & 1.77 & 1.77 & 1.97 \\
\hline $\operatorname{CCSD}(\mathrm{T})$ & 0.02 & 0.91 & -0.72 & 0.73 & 0.45 & 0.47 & 0.75 \\
\hline CR-CC $(2,3), A$ & 0.42 & 1.04 & -0.48 & 0.56 & 0.68 & 0.68 & 0.78 \\
\hline CR-CC(2,3), B & 0.60 & 1.12 & -0.30 & 0.44 & 0.72 & 0.72 & 0.77 \\
\hline CR-CC(2,3), C & 0.40 & 0.90 & -1.10 & 1.10 & 0.69 & 0.69 & 0.94 \\
\hline CR-CC $(2,3), \mathrm{D}$ & 0.40 & 0.90 & -1.10 & 1.10 & 0.68 & 0.68 & 0.94 \\
\hline \multicolumn{8}{|c|}{ aug-cc-pV(T+d)Z } \\
\hline CCSD & 2.57 & 2.57 & 1.51 & 1.51 & 1.71 & 1.71 & 1.98 \\
\hline $\operatorname{CCSD}(\mathrm{T})$ & -0.19 & 0.54 & -0.50 & 0.64 & 0.38 & 0.38 & 0.55 \\
\hline CR-CC $(2,3), A$ & 0.21 & 0.56 & -0.25 & 0.48 & 0.61 & 0.61 & 0.54 \\
\hline CR-CC(2,3), B & 0.40 & 0.64 & -0.08 & 0.35 & 0.66 & 0.66 & 0.53 \\
\hline CR-CC(2,3), C & 0.16 & 0.41 & -0.85 & 0.85 & 0.62 & 0.62 & 0.63 \\
\hline CR-CC(2,3), D & 0.17 & 0.41 & -0.84 & 0.84 & 0.62 & 0.62 & 0.63 \\
\hline \multicolumn{8}{|c|}{ aug-cc-pCVTZ } \\
\hline CCSD(full) & 2.77 & 2.77 & 1.75 & 1.75 & 1.63 & 1.63 & 2.13 \\
\hline $\operatorname{CCSD}(\mathrm{T})($ full) & -0.12 & 0.51 & -0.30 & 0.59 & 0.23 & 0.23 & 0.49 \\
\hline CR-CC(2,3), A(full) & 0.30 & 0.55 & -0.03 & 0.40 & 0.46 & 0.46 & 0.48 \\
\hline CR-CC (2,3), B(full) & 0.49 & 0.64 & 0.15 & 0.32 & 0.51 & 0.51 & 0.48 \\
\hline CR-CC(2,3), C(full) & 0.25 & 0.40 & -0.57 & 0.69 & 0.47 & 0.47 & 0.53 \\
\hline CR-CC(2,3), D(full) & 0.25 & 0.40 & -0.57 & 0.69 & 0.47 & 0.47 & 0.53 \\
\hline \multicolumn{8}{|c|}{ aug-cc-pCV $(T+d) Z$} \\
\hline CCSD(full) & 2.75 & 2.75 & 1.80 & 1.80 & 1.64 & 1.64 & 2.15 \\
\hline $\operatorname{CCSD}(\mathrm{T})($ full) & -0.14 & 0.46 & -0.26 & 0.57 & 0.24 & 0.24 & 0.46 \\
\hline
\end{tabular}

